\title{
High Prevalence of Multidrug Resistant Klebsiella Species Isolated from the Yaounde University Teaching Hospital, Cameroon
}

\author{
Emilia Enjema Lyonga Mbamyah ${ }^{1,2 *}$, Florence Anjabie Enyeji1, Judith Torimiro', \\ Patience Mangum ${ }^{3}$, Modestine Djuissi', Aime-Caesar Teukam1, Martha Tongo Mesembe ${ }^{2}$, \\ George Mondinde Ikomey, ${ }^{1,2}$, Anicette Chafa Betbeui ${ }^{4}$, Dieudonné Sedena ${ }^{5}$, William Baiye ${ }^{4}$, \\ Agnes Bedie Eyoh², Hortense Kamga Gonsu ${ }^{1,4}$
}

\author{
${ }^{1}$ Faculty of Medicine and Biomedical Sciences, The University of Yaounde 1, Yaounde, Cameroon \\ ${ }^{2}$ Center for the Study and Control of Communicable Diseases, Faculty of Medicine and Biomedical Sciences, The University of \\ Yaounde 1, Yaounde, Cameroon \\ ${ }^{3}$ Department of Microbiology, School of Health Sciences, Catholic University of Central Africa, Yaounde, Cameroon \\ ${ }^{4}$ Yaounde University Teaching Hospital, Yaounde, Cameroon \\ ${ }^{5}$ Faculty of Science, University of Buea, Buea, Cameroon \\ Email: ^emilialyo@yahoo.co.uk
}

How to cite this paper: Mbamyah, E.E.L. Enyeji, F.A., Torimiro, J., Mangum, P., Djuissi, M., Teukam, A.-C., Mesembe, M.T., Ikomey, G.M., Betbeui, A.C., Sedena, D., Baiye, W., Eyoh, A.B. and Gonsu, H.K. (2021) High Prevalence of Multidrug Resistant Klebsiella Species Isolated from the Yaounde University Teaching Hospital, Cameroon. Open Journal of Medical Microbiology, 11, 91-99. https://doi.org/10.4236/ojmm.2021.112008

Received: March 13, 2021

Accepted: June 1, 2021

Published: June 4, 2021

Copyright () 2021 by author(s) and Scientific Research Publishing Inc. This work is licensed under the Creative Commons Attribution International License (CC BY 4.0).

http://creativecommons.org/licenses/by/4.0/

\section{(c) (i) Open Access}

\begin{abstract}
Background and Purpose: Klebsiella species are amongst the most common causes of a variety of community-acquired and hospital-acquired infections (HAI), characterized by high morbidity and mortality rates. Most infections caused by Klebsiella species are usually treated using antibiotics. The aim of this study was to determine the antimicrobial resistance profile of Klebsiella species isolated from in-patients and out-patients at the Yaounde University Teaching Hospital. The data generated will go a long way to improve on the choice of an adequate empiric antibiotic treatment for infections caused by Klebsiella species. Methodology: A cross-sectional descriptive study was carried out over a period of 6 months, spanning from February 2019 to July 2019 with a sample size of 37 isolates, obtained from 6 different clinical specimens. Identification of isolates was done using API 20E identification system (Biomerieux SA, Lyon, France). Susceptibility to antibiotics was tested as described by Kirby-Bauer in 1956. Inhibition diameters were interpreted according to recommendations from the European Committee on Antimicrobial Susceptibility Testing (EUCAST, 2019). Results and Conclusion: Among the 37 Klebsiella isolates identified, Klebsiella pneumoniae was the most prevalent species isolated with a percentage of $54.1 \%$, followed by Klebsiella rhinoscleromatis $18.9 \%$, Klebsiella ozaenae $16.2 \%$ and Klebsiella oxytoca, $10.8 \%$. The resistance pattern of Klebsiella to amoxicillin, amoxicillin/clavulanate, tircacil-
\end{abstract}


lin, tircacillin + clavulanic acid, piperacillin, piperacillin + tazobactam, cefalotin, cefuroxim, ceftazidime, cefotaxime, ceftriaxone, cefepime, imipenem, meropenem, aztreonam, amikacin, gentamicin, tobramycin, trimethoprim/ sulfamethoxazole, nalidixic acid, pipemidic acid, norfloxacin, ciprofloxacin, levofloxacin, ofloxacin, and moxifoxacin was as follows; $100 \%, 86.5 \%, 97.3 \%$, $83.6 \%, 86.5 \%, 16.2 \%, 86.5 \%, 83.8 \%, 78.4 \%, 32.4 \%, 78.4 \%, 76.7 \%, 2.7 \%, 2.7 \%$, $76.7 \%, 13.5 \%, 75.7 \%, 73.0 \%, 91.9 \%, 51.4 \%, 48.6 \%, 64.9 \%, 48.6 \%, 48.6 \%, 73.0 \%$ and $62.2 \%$ respectively. Multidrug resistance was observed in $94.6 \%$ of the Klebsiella isolates. Conclusion: This study shows that the level of multidrug resistance is high. The isolates expressed good sensitivity to carbapenems, piperacillin + tazobactam, amikacin and high resistance to all other antimicrobials tested. Therefore, antimicrobial susceptibility testing prior to prescriptions should be encouraged and sensitization of the population about consequences of inappropriate antibiotic treatment and auto medication should be enforced as a means to curb antimicrobial resistance.

\section{Keywords}

Klebsiella Species, Antimicrobial Resistance, Multidrug Resistance

\section{Introduction}

Klebsiella species are found in nature in water, soil and animals and they can colonize medical devices and the healthcare environment [1] [2]. Klebsiella species are among the most common causes of a variety of community-acquired and hospital-acquired infections (HAIs). These diseases cause an increase in morbidity and mortality [3].

They are considered opportunistic pathogens colonizing mucosal surfaces without causing pathology; however, from mucosae Klebsiella may disseminate to other tissues causing life-threatening infections including bronchopneumonia, urinary tract infections (UTIs), bloodstream infections and sepsis [4].

Most infections caused by Klebsiella species are usually treated using antibiotics. The high rate at which antibiotics are used all around the world in human therapy and in veterinary medicine has given rise to antimicrobial resistance [5]. Antimicrobial resistance is associated with increased patient morbidity and mortality and contributes to escalating health care cost and prolonged stay in hospital [6] [7].

Today, antimicrobial resistance is a global public health problem [8] [9]. The aim of this study was to determine the antimicrobial resistance profile of Klebsiella species isolated from the Yaounde University Teaching Hospital. This data will be useful in choosing an adequate antibiotic treatment for infections caused by Klebsiella species.

\section{Methodology}

A cross sectional descriptive study was carried out for 6 months, spanning from 
February to July 2019. Isolates were collected from 6 different clinical specimens: blood, pus, wound, urinary catheter, venous catheter and urine. The isolates were from in-patients and out-patients consulted at the Yaounde University Teaching Hospital. These specimens were cultured on eosin methylene blue agar (EMB) and incubated at $37^{\circ} \mathrm{C}$ for $18-24$ hours. Based on colony morphology, suspected Klebsiella spp. colonies were identified using API 20E identification kits (Biomerieux SA, Lyon, France). Klebsiella species were identified from 37 specimens. Antimicrobial susceptibility testing using the Kirby-Bauer disc diffusion method was done following recommendations from the European Committee on Antimicrobial Susceptibility Testing (EUCAST 2019) [10] and inhibition diameters were reported.

The 26 antibiotics tested were from the following classes: quinolones, penicillins, cephalosporins, monobactams, carbapenems, aminoglycosides and sulphonamides. Seven quinolones were tested namely: nalidixic acid $(30 \mu \mathrm{g})$, pipemidic acid $(20 \mu \mathrm{g})$, norfloxacin $(5 \mu \mathrm{g})$, ciprofloxacin $(10 \mu \mathrm{g})$, levofloxacin $(5 \mu \mathrm{g})$ ofloxacin $(5 \mu \mathrm{g})$ and moxifoxacin $(5 \mu \mathrm{g})$. The penicillins tested were: amoxicillin (25 $\mu \mathrm{g})$, amoxicillin/clavulanate $(20 / 10 \mu \mathrm{g})$, tircacillin $(75 \mu \mathrm{g})$, tircacillin + clavulanic acid $(75 / 10 \mu \mathrm{g})$, piperacillin $(30 \mu \mathrm{g})$, and piperacillin + tazobactam $(30 / 6 \mu \mathrm{g})$. Cephalosporins tested included: cefalotin $(30 \mu \mathrm{g})$, cefuroxime $(30 \mu \mathrm{g})$, ceftriaxone $(5 \mu \mathrm{g})$, ceftazidime $(10 \mu \mathrm{g})$, cefotaxime $(5 \mu \mathrm{g})$, and cefepime (30 $\mu \mathrm{g})$. Among the carbapems imipenem $(10 \mu \mathrm{g})$ and meropenem $(10 \mu \mathrm{g})$ were tested. The following aminoglycosides were tested: amikacin $(30 \mu \mathrm{g})$, gentamicin (10), and tobramycin $(10 \mu \mathrm{g})$. One sulphonamide: trimethoprim/sulfamethoxazole $(1.25 / 23.75 \mu \mathrm{g})$ was tested and aztreonam $(30 \mu \mathrm{g})$ was the only monobactam tested. Quality control of antibiotic discs was done using Escherichia coli ATCC 25922. Data analysis was carried out using Microsoft Excel 2016 and SPSS version 25.

\section{Results}

Thirty seven Klebsiella isolates were identified using the API 20E identification system. Among the species isolated Klebsiella pneumoniae was the most prevalent with $54.1 \%$, followed by Klebsiella rhinoscleromatis $18.9 \%$, Klebsiella ozaenae $16.2 \%$ and Klebsiella oxytoca, $10.8 \%$ as shown on Figure 1 below.

Klebsiella species were most isolated from pus $24.3 \%$, urinary catheter and urine with a frequency of $21.6 \%$ each and isolated least from venous catheter $5.4 \%$ as shown on Table 1 .

The antimicrobial resistance profile of the Klebsiella species to all the antibiotics tested according to the European Committee on Antimicrobial Susceptibility Testing (EUCAST 2019) [10] standards are represented on Figure 2.

The natural resistance of Klebsiella species to ampicillin was confirmed with a $100 \%$ resistance. The isolates were most resistant to tircacillin $(97.3 \%)$ and sulfamethoxazole + trimethoprim (91.9\%). The isolates showed the least resistance to imipenem and meropenem with $2.7 \%$ each as shown on Figure 2.

Multidrug resistance was observed in $94.6 \%$ of the Klebsiella isolates as shown on Figure 3. No wild type was found. 


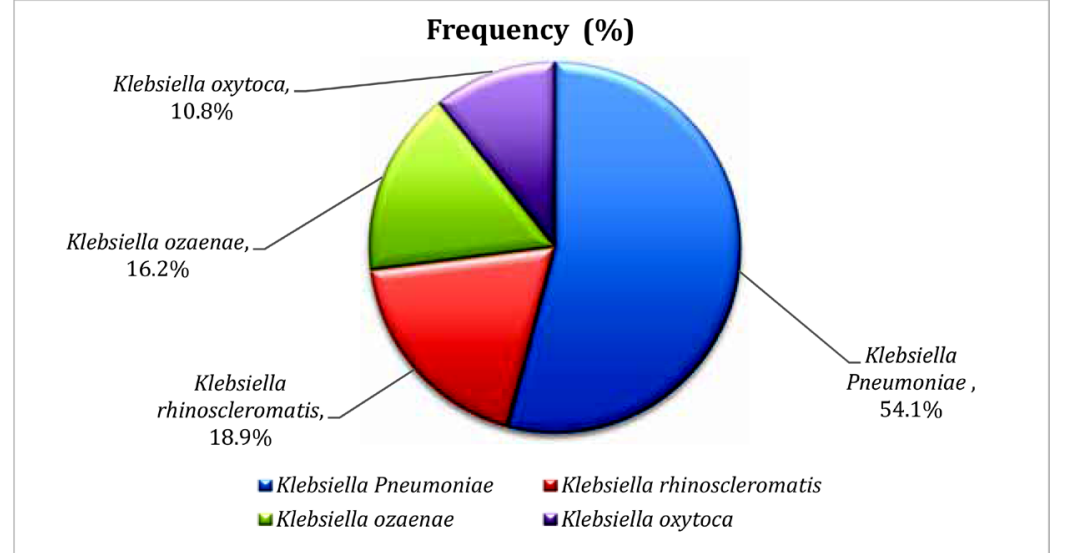

Figure 1. Distribution of Klebsiella species isolated.

Table 1. Distribution of clinical specimens.

\begin{tabular}{ccc}
\hline Specimen & Number of subjects & percentage \\
\hline Pus & 9 & $24.3 \%$ \\
Urinary catheter & 8 & $21.6 \%$ \\
Urine & 8 & $21.6 \%$ \\
Blood culture & 7 & $18.9 \%$ \\
Wound & 3 & $8.1 \%$ \\
Venous catheter & 2 & $5.4 \%$ \\
Total & 37 & 100 \\
\hline
\end{tabular}

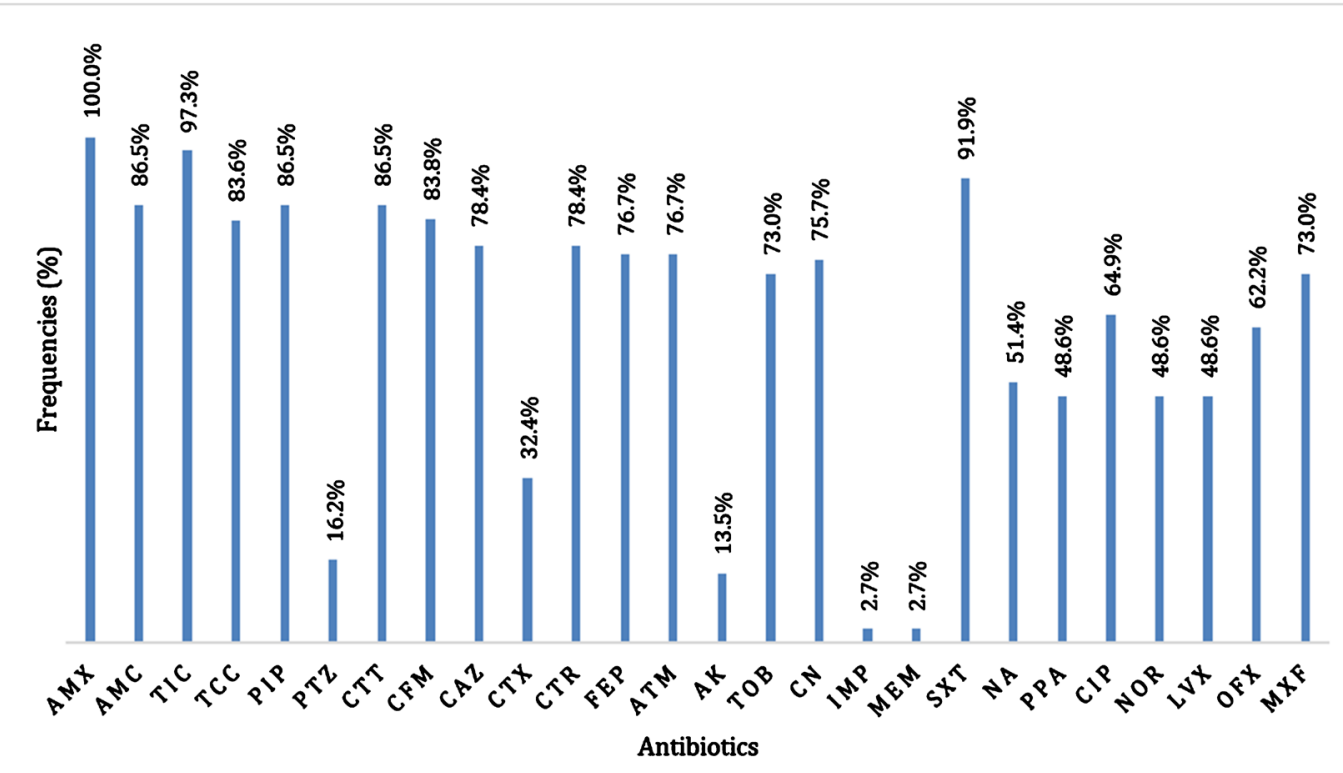

AMX: Amoxicillin; AMC: Amoxicillin + clavulanic acid; TIC: Ticarcillin; TCC: Ticarcillin + clavulanic acid; PIP: Piperacillin; PTZ: Piperacellin + tazobactam; CTT: Cefalotin; CFM: Cefuroxime; CAZ: Ceftazidime; CTX: Cefotaxime; CTR: Ceftriaxone; FEP: Cefepime; IPM: Imipenem; MEM: Meropenem; AK: Amikacin; TOB: Tobramycin; CN: Gentamicin; SXT: Sulfamethoxazole + trimethoprim; ATM: Aztreonam; NA: Nalidixic acid; PPA: Pipemidic acid; NOR: Norfloxacin; CIP: Ciprofloxacin; LVX: Levofloxacin; OFX: Ofloxacin; MXF: Mixofloxacin.

Figure 2. Antimicrobial resistance profile of the Klebsiella spp. 


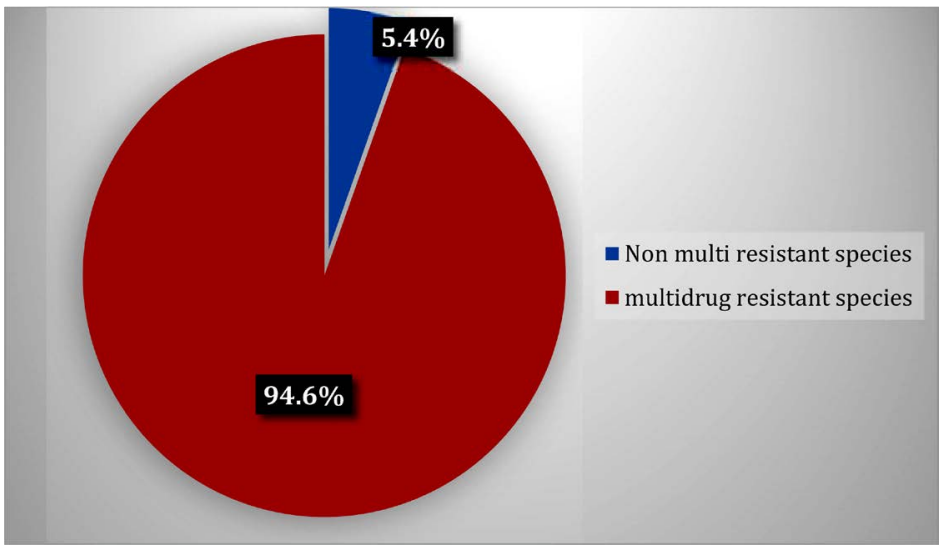

Figure 3. Multidrug resistant Klebsiella species.

\section{Discussion}

In this study the majority of isolates were Klebsiella pneumoniae 20/37 (54.1\%). This trend is similar to previous work by Sunilkumar and collaborators in India (98\%) [11] and Hansel and collaborators in the United Kingdom (76\%) [12]. The frequency of Klebsiella pneumoniae confirms that it is one of the leading causes of infections [13].

The isolates were from 6 different types of clinical specimens: blood, pus, urinary catheter, urine, venous catheter, and wound. Klebsiella species were most isolated from pus (24.3\%), a result similar to a study carried out in Algeria.

The isolates were resistant to all penicillins tested. Resistance to amoxicilin + clavulanic acid was high, 32/37 (86.5\%), likewise to tircacillin 36/37 (97.3\%), tircacillin + clavullanic acid 31/37 (83.6\%) and piperacillin 32/37 (86.5\%). However, the antimicrobial activity of piperacillin was improved with the combination of piperacillin with tazobactam resulting in a reduction of the rate of resistance of the isolates $6 / 37$ (16.2\%). All the isolates were resistant to amoxicilin 37/37 (100\%), which was similar to that obtained by Arafat et al., 2009 who in Algeria recorded 50/50 (100\%) resistance to Amoxicillin [14]. Klebsiella spp. has a natural resistance to amoxicillin due to the presence of a Class A SHV-1 penicillinase [15].

This overall high rate of resistance of the isolates to the penicillins could be explained by the over use of these drugs in the treatment of common infections and ease of drug acquisition without prescription and even from road side vendors.

The resistance of the isolates to the cephalosporins was also very high with the following frequency recorded: cefalotine $32 / 37$ (86.5\%), cefuroxime $31 / 37$ (83.8\%), ceftazidime 29/37 (78.4\%), ceftriazone 29/37 (78.4\%) and cefepime 28/37 (76.7\%). The least resistance of the isolates was to cefotaxime 12/37 (32.4\%). This result is different from results reported by Betbeui and collaborators in 2015 from three referral hospitals in Yaounde. The study reported lower rates of resistance for the cephalosporins [16]. Thus there has been an increase over time in resistance to cephalosporins. The resistance of isolates to cephalosporins is as a result of the production of extended spectrum betalactamases that hydrolyze penicillins, 
first, second, third, fourth generation cephalosporins and monobatams.

The resistance of the isolates to aztreonam was high 28/37 (76.7\%). This result is similar to results had by Gangoue-Pieboji et al. 2006 who in Yaounde recorded $75 \%$ resistance to aztreonam [17]. This shows that resistance has remained high over time.

The resistance to carbapenems was low for both imipenem and meropenem $1 / 37$ (2.7\%). This result is similar to results by Gangoue-Pieboji et al., who in Yaounde had 2\% resistance to carbapenems [17]. The low resistance of the isolates to these antibiotics could be explained by the fact that these drugs are reserved as last line treatment, they are expensive and they can be administered only via the intravenous route [18].

The isolates expressed high resistance to the aminoglycosides such as tobramycin 27/37 (73.0\%) and gentamicin 28/37 (75.7\%) with least resistance recorded to amikacin 5/37 (13.5\%). Again there is a general increase in resistance (except for resistance to amikacin) compared to results by Gangoue-Pieboji et al., 2006, who reported a resistance of 35\% [17].

The isolates expressed high resistance to sulphamethoxazole + trimetoprim 34/37 (91.9\%). This frequency is higher than results obtained by Arafa et al., 2009 who in Algeria reported 73\% of their isolates were resistant to sulphometoxazole + trimetoprim [14]. This antibiotic is often used for the treatment of UTI's but the high resistance rate recorded in this study, disqualifies it for such use.

Among the quinolones, the isolates were most resistant to moxifloxacin 27/37 (73.0\%), ciprofloxacin 24/37 (64.9\%), ofloxacin 23/37 (62.2\%) and nalidixic acid 19/37 (51.4\%). The isolates were least resistant to pipemidic acid, norfloxacin and levofloxacin 18/37 (48.6\%) each. This result is different from results had by Chakraboty et al., 2016 who recorded low levels of resistance to quinolones [19]. Resistance to ciprofloxacin is considered resistance to all quinolones because of acquisition of at least two mutations in either $g y r \mathrm{~A}$ or $g y r \mathrm{~B}$ and $\operatorname{par}^{\mathrm{C}} \mathrm{C}$. These genes are capable of mediating low-level quinolone resistance that causes high-level resistance to arise in the presence of quinolones at therapeutic levels [20].

The emergence of multidrug resistant Klebsiella species has become a major public health concern worldwide and has been associated with outbreaks of infections in developing countries due to the indiscriminate use of antibiotics [21]. An isolate is said to be multidrug resistant if it is resistant to three or more antimicrobial classes. We found that $95 \%$ of the isolates were multidrug resistant species. Multidrug resistance in Klebsiella species varies in different parts of the world. In India, 54\% was reported [22] and in Nigeria 75.8\% [23]. The high level of multidrug resistance in this study could be due to an interplay of other resistance mechanisms co-expressed by the isolates such as extended spectrum betalactamases, quinolone resistance genes and $a a c\left(6^{\prime}\right)-I b-c r$ enzymes which hydrolyze quinolones and aminoglycosides. Furthermore, prior antibiotic use in hospitals or through auto-medication, overuse of antibiotics in livestock and fish farming, poor infection control in health care facilitates and poor hygiene and 
sanitation exercebates multidrug resistance.

This study however had some limitations. The sample size was small as isolates were collected from only one hospital, the Yaounde University Teaching Hospital. This study needs to be extended to other hospitals in Yaounde for a longer period to better understand resistance trends in Yaounde. For the purpose of infection control and clinical relevance of the results, it would be necessary for the resistance phenotypes to be characterized.

\section{Conclusion}

This study shows that the level of multidrug resistance is high. The isolates expressed good sensitivity to the carbapenems, piperacillin + tazobactam, amikacin and high resistance to all other antimicrobials tested. This indicates that with the exception of a few antibiotics, commonly used affordable antibiotics may not treat infections caused by Klebsiella spp. Therefore, antimicrobial susceptibility testing prior to prescriptions should be encouraged and sensitization of the population about the consequences of antibiotic abuse and auto medication should be enforced as a means to curb antimicrobial resistance. The surveillance of antimicrobial resistance should be put in place to monitor clinically relevant isolates.

\section{Acknowledgements}

This work was carried out with the aid of a grant from UNESCO and the International Development Research Center (IDRC), Ottawa, Canada. The views expressed herein do not necessarily represent those of UNESCO, IDRC or its Board of Governors.

The authors wish to thank the hospital personnel at the Bacteriology Unit of the Yaounde University Teaching for the services offered during the period of specimen collection and the staff of the Centre for the Study and Control of Communicable Diseases (CSCCD), Faculty of Medicine and Biomedical Sciences, the University of Yaoundé 1.

\section{Authors' contributions}

Emilia Enjema Lyonga Mbamyah conceived the study and designed it together with Florence Anjabie Enyeji, Judith Torimiro and Hortense Kamga Gonsu. Emilia Enjema Lyonga Mbamyah, Florence Anjabie Enyeji, Modestine Djuissi, Patience Mangum, and Dieudonné Sedena, Aime-Caesar Teukam, Agnes Bedie Eyoh, William Baiye conducted the laboratory aspect of the study with contributions from Martha Tongo Mesembe and George Mondinde Ikomey. The general supervision was carried out by Emilia Enjema Lyonga Mbamyah. Emilia Enjema Lyonga Mbamyah drafted the article with contributions from Florence Anjabie Enyeji ${ }^{1}$ and Martha Tongo Mesembe. All the authors reviewed the article. All the authors read and agreed to the final manuscript. 


\section{Conflicts of Interest}

The authors declare no conflicts of interest regarding the publication of this paper.

\section{References}

[1] Podschun, R. and Ullmann, U. (1998) Klebsiella spp. as Nosocomial Pathogens: Epidemiology, Taxonomy, Typing Methods, and Pathogenicity Factors. Clinical Microbiology Reviews, 11, 589-603. https://doi.org/10.1128/CMR.11.4.589

[2] Podschun, R., Pietsch, S., Höller, C. and Ullmann, U. (2001) Incidence of Klebsiella Species in Surface Waters and Their Expression of Virulence Factors. Applied and Environmental Microbiology, 67, 3325-3327. https://doi.org/10.1128/AEM.67.7.3325-3327.2001

[3] Tian, L.J., Tan, R.M., Chen, Y., Sun, J.Y., Liu, J.L., Qu, H.P. and Wang, X.L. (2016) Epidemiology of Klebsiella pneumoniae Bloodstream Infections in a Teaching Hospital: Factors Related to the Carbapenem Resistance and Patient Mortality. Antimicrobial Resistance and Infection Control, 5, 48. https://doi.org/10.1186/s13756-016-0145-0

[4] Bengoechea, J.A. and Sa Pessoa, J. (2019) Klebsiella pneumoniae Infection Biology: Living to Counteract Host Defences. FEMS Microbiology Review, 43, 123-144. https://doi.org/10.1093/femsre/fuy043

[5] Basode, V.K., Abdulhaq, A., Alamoudi, M.U., Tohari, H.M., Quhal, W.A. and Madkhali, A. (2018) Prevalence of a Carbapenem-Resistance Gene (KPC), Vancomycin-Resistance Genes (van A/B) and a Methicillin-Resistance Gene (mecA) in Hospital and Municipal Sewage in a Southwestern Province of Saudi Arabia. BMC Research Notes, 11, 30. https://doi.org/10.1186/s13104-018-3167-2

[6] Adeosun, I.J., Oladipo, E.K., Ajibade, O.A., Olotu, T.M., Oladipo, A.A., Awoyelu, E.H., Alli, O.A.T. and Oyawoye, O.M. (2019) Antibiotic Susceptibility of Klebsiella pneumoniae Isolated from Selected Tertiary Hospitals in Osun State, Nigeria. Iraqi Journal of Science, 60, 1423-1429.

[7] Kumar, A.R. (2013) Antimicrobial Sensitivity Pattern of Klebsiella Pneumoniae Isolated from Sputum from Tertiary Care Hospital, Surendranagar, Gujarat and Issues Related to the Rational Selection of Antimicrobials. Scholars Journal of Applied Medical Sciences, 1, 928-933.

[8] Wise, R., Hart, T., Cars, O., Streuleus, M. and Helmut, H.P. (1998) Antimicrobial Resistance Is a Major Threat to Public Health. British Medical Journal, 317, 609-610. https://doi.org/10.1136/bmj.317.7159.609

[9] Shaes, D.M., Gerdin, D.N., John, J.F., Craig, W.A., Borstein, D.L., Ducan, R.A., et al. (1997) Society for Healthcare Epidemiology of America and Infectious Diseases of America Joint Committee on the Prevention of Antimicrobial Resistance. Clinical Infectious Disease, 25, 584-599. https://doi.org/10.1086/513766

[10] European Committee on Antimicrobial Susceptibility Testing and Standards (EUCAST) for Antimicrobial Susceptibility Testing January 2019 V1.0.

[11] Sunilkumar, B. and Roopa, C. (2015) Isolation and Antibiogram of Klebsiella Species from Various Clinical Specimens. International Journal of Current Microbiology and Applied Sciences, 4, 991-995.

[12] Hansen, D.S., Gottschau, A. and Kolmos, H.J. (1998) Epidemiology of Klebsiella Bacteraemia: A Case Control Study Using Escherichia coli Bacteraemia as Control. Journal Hospital Infection, 38, 119-132. 
https://doi.org/10.1016/S0195-6701(98)90065-2

[13] Monnet, D., Freney, J., Brun, Y., Boeufgras, J.M. and Fleurette, J. (1991) Difficulties in Identifying Klebsiella Strains of Clinical Origin. International Journal of Medical Microbiology, 274, 456-464. https://doi.org/10.1016/S0934-8840(11)80081-2

[14] Arafa, N., Smati, F., Schefte, J.M. and Meunier, O. (2009) Characterization Phenotypique et genotypique de souches des Klebsiella pneumonia subsp pneumonia isolees a l'hopital Universitaire de Constantine, Algeria. Science and Technology Journal, 30, 43-49.

[15] Wyres, K. and Holt, K. (2018) Klebsiella pneumoniae as a Key Trafficker of Drug Resistance from the Environment to Clinically Important Bacteria. Current Opinion in Microbiology, 45, 131-139. https://doi.org/10.1016/j.mib.2018.04.004

[16] Betbeui, C.A., Kamga, G.H., Toukam, M., Mbakop, D.C., Lyonga, E.E., Bilong, S. and Koulla-Shiro, S. (2015) Phenotypic Detection of Extended Spectrum Beta Lactamases and Carbapenemases Produced by Klebsiella spp. Isolated from Three Referral Hospitals in Yaounde Cameroon. British Microbiology Research Journal, 9, 1-9. https://doi.org/10.9734/BMRJ/2015/18250

[17] Gangoue-Pieboji, J., Koulla-Shiro, S., Ngassam, P., Adiogo, D. and Ndumbe, P. (2006) Antimicrobial Activity against Gram Negative Bacilli from Yaounde Central Hospital Cameroon. African Health Science, 6, 232-235.

[18] Meletis, G. (2016) Carbapenem Resistance; Overview of the Problem and Future Perspectives. Therapeutic Advances in Infectious Disease, 3, 15-21.

https://doi.org/10.1177/2049936115621709

[19] Chakraborty, S., Mohsina, K., Sarker, P.K., Alam, M.D.Z., Karim, M.A. and Sayem, S.M.A. (2016) Prevalence, Antibiotic Susceptibility Profiles and ESBL Production in Klebsiella pneumoniae and Klebsiella oxytoca among Hospitalized Patients. Periodicum Biologorum, 118, 53-58. https://doi.org/10.18054/pb.2016.118.1.3160

[20] Jacoby, G., Walsh, K., Mills, D., Walker, V. and Oh, H. (2006) qnrB, Another Plasmid-Mediated Gene for Quinolone Resistance. Antimicrobial Agents and Chemotherapy, 50, 1178-1182. https://doi.org/10.1128/AAC.50.4.1178-1182.2006

[21] Akter, J., Chowdhury, A.M.M.A. and Forkan, M.A. (2014) Study on Prevalence and Antibiotic Resistance Pattern of Klebsiella Isolated from Clinical Samples in South East Region of Bangladesh. American Journal of Drug Discovery and Development, 4, 73-79. https://doi.org/10.3923/ajdd.2014.73.79

[22] Sikarwar, A.S. and Batra, H.V. (2011) Prevalence of Antimicrobial Drug Resistance of Klebsiella pneumoniae in India. International Journal of Bioscience, Biochemistry and Bioinformatics, 1, 211-215. https://doi.org/10.7763/IJBBB.2011.V1.38

[23] Chikwendu, C.I., Amadi, E.S. and Obi, R.K. (2010) Prevalence and Antimicrobial Resistance in Pseudomonas aeruginosa and Klebsiella pneumoniae Isolates from Non-Clinical Urine Samples. New York Science Journal, 3, 194-200. 